\title{
Halide and Thiocyanate Metal Acid Complexes Extraction in the Water - Ethoxylated Nonylphenol - Ammonium Sulfate System
}

\author{
Anastasya V. Stankova ${ }^{a, b}$, \\ Aleksandr M. Elokhov ${ }^{\mathrm{b}, \mathrm{c}}$ and Andrey E. Lesnov ${ }^{\mathrm{a}, \mathrm{d}}$ * \\ ${ }^{a}$ Institute of Technical Chemistry of the UB RAS \\ 3 Koroleva Str., Perm, 614013, Russia \\ ${ }^{b}$ Perm State University \\ 15 Bukireva Str., Perm, 614990, Russia \\ ${ }^{c}$ Natural Science Institute of Perm State University \\ 4 Genkel Str., Perm, 614990, Russia \\ ${ }^{d}$ Perm State Agrarian - Technological University \\ 23 Petropavlovskaia Str., Perm, 614990, Russia
}

Received 04.02.2019, received in revised form 09.07.2019, accepted 22.08.2019

\begin{abstract}
Nonionic ethoxylated surfactants, including ethoxylated nonylphenols, can be considered as neutral oxygen-containing extraction reagents, the formation of delamination systems with which is possible when salting-out with inorganic salts. In this work, the distribution of halide and thiocyanate acid complexes of thallium (III), iron (III), indium and gallium in the water - ethoxylated nonylphenol (neonol AF 9-12) - ammonium sulfate system at $25^{\circ} \mathrm{C}$ was investigated. It is established that thallium (III) is quantitatively extracted in the form of tetrahalidetallate-ion with an acidity of more than 0.1 mol/l, extraction of other metals is not quantitative. Among the thiocyanate acid complexes, zinc, cobalt and copper (II) are quantitatively concentrated, which can be used for group concentration of these metals or their extraction-spectrophotometric determination methods.
\end{abstract}

Keywords: liquid-liquid extraction, ethoxylated nonylphenols, halide acid complexes, surfactants.

Citation: Stankova A.V., Elokhov A.M., Lesnov A.E. Halide and thiocyanate metal acid complexes ex-traction in the water ethoxylated nonylphenol - ammonium sulfate system, J. Sib. Fed. Univ. Chem., 2019, 12(3), 328-335. DOI: 10.17516/19982836-0130.

(C) Siberian Federal University. All rights reserved

* Corresponding author E-mail address: elhalex@yandex.ru 


\title{
Экстракция галогенидных и тиоцианатных \\ ацидокомплексов металлов в системе вода - \\ оксиэтилированный нонилфенол - сульфат аммония
}

\author{
А.В. Станкова ${ }^{\mathrm{a}, \boldsymbol{\sigma}}$, А.М. Елохов ${ }^{\text {б,в }}$, А.Е. Леснов ${ }^{\mathrm{a}, \mathrm{r}}$ \\ ${ }^{a}$ Институт технической химии УО РАН \\ Россия, 614013, Пермь, ул. Королева, 3 \\ ${ }^{6}$ Пермский государственный \\ национальный исследовательский университет, \\ Россия, 614990, Пермь, ул. Букирева, 15 \\ ${ }^{8}$ Естественнонаучный институт \\ Пермского государственного \\ национального исследовательского университета \\ Россия, 614990, Пермь, ул. Генкеля, 4 \\ ¿Пермский государственный аграрно-технологический университет \\ Россия, 614990, Пермь, ул. Петропавловская, 23.
}

Неионные оксиэтилированные ПАВ, в том числе оксиэтилированные нонилфенолы, могут рассматриваться как нейтральные кислородсодержащие экстракиионные реагенты, образование расслаивающихся систем с которыми возможно при высаливании неорганическими солями. В работе исследовано распределение галогенидных и тиоиианатных ацидокомплексов таллия (III), железа (III), индия и галлия в системе вода-оксиэтилированный нонилфенол (неонол АФ 9-12) - сульфат аммония при $25^{\circ} \mathrm{C}$. Установлено, что количественно извлекается таллий в виде тетрагалогенидталлат-иона при кислотности более 0,1 моль/л, извлечение остальных металлов не количественное. Среди тиочианатных ацидокомплексов количественно концентрируется ицин, кобальт и медь (II), что может использоваться для группового концентрирования указанных металлов или их экстракичионо-спектрофотометрического определения.

Ключевые слова: жидкостная экстракция, оксиэтилированные нонилфеноль, галогенидные ацидокомплексы, поверхностно-активные вещества.

\section{Введение}

Задача повышения безопасности экстракционных процессов решается путем исключения токсичных, летучих и пожароопасных органических растворителей. В качестве перспективных нетрадиционных растворителей рассматривают водорастворимые полимеры [1-3], поверхностно-активные вещества [4-6], ионные жидкости [7-9] и глубоко эвтектические смеси [10-12]. Доступность, низкая стоимость, высокая степень биоразлагаемости, а также широкий спектр выпускаемых ПАВ обуславливают высокий интерес к поиску новых методов экстракции веществ различной природы в системах на основе неионных $[13,14]$, катионных $[15,16]$, анионных $[17,18]$ ПАВ или их смесей $[19,20]$. Наибольшее распространение в экстракции по- 
лучили неионные оксиэтилированные ПАВ в силу возможности варьирования степени оксиэтилирования и, соответственно, их способности высаливания при действии неорганических солей или изменения температуры. Наличие в растворе анионов-комплексообразователей, таких как галогенид- или тиоцианат-ионы, приводит к образованию ацидокомплексов с ионами металлов, которые экстрагируются протонированными формами оксиэтилированных ПАВ по анионообменному механизму [21].

Ранее нами были исследованы закономерности высаливания технических оксиэтилированных нонилфенолов (неонолов) $[22,23]$ и изучено распределение хлоридных ацидокомплексов катионов металлов в присутствии различных высаливателей [24]. Настоящая работа посвящена изучению экстракции ионов металлов в присутствии галогенид- и тиоциант-ионов в системе вода - неонол АФ-9-12 - вода.

\section{Методика эксперимента}

В работе использованы: неионное оксиэтилированное ПАВ неонол АФ-9-12 (ТУ 2483-07705766801-98, оксиэтилированный нонилфенол, $\mathrm{C}_{9} \mathrm{H}_{19} \mathrm{C}_{6} \mathrm{H}_{4} \mathrm{O}\left(\mathrm{C}_{2} \mathrm{H}_{4} \mathrm{O}\right)_{12} \mathrm{H}$, основное вещество не менее $98 \%$, вод не более $0,5 \%$, полиэтиленгликоль не более $1,5 \%$ ); $\mathrm{CuSO}_{4} \cdot 5 \mathrm{H}_{2} \mathrm{O}, \mathrm{CoSO}_{4} \cdot 7 \mathrm{H}_{2} \mathrm{O}$, $\mathrm{CdSO}_{4} \cdot \mathrm{H}_{2} \mathrm{O}, \mathrm{ZnSO}_{4} \cdot 7 \mathrm{H}_{2} \mathrm{O}, \mathrm{Fe}_{2}\left(\mathrm{SO}_{4}\right)_{3}, \mathrm{Tl}_{2}\left(\mathrm{SO}_{4}\right)_{3}, \mathrm{Ga}_{2}\left(\mathrm{SO}_{4}\right)_{3}, \mathrm{In}_{2}\left(\mathrm{SO}_{4}\right)_{3}$ квалификации х.ч.; $\mathrm{HCl}, \mathrm{H}_{2} \mathrm{SO}_{4}$, $\mathrm{KI}, \mathrm{KSCN}, \mathrm{KBr}$ квалификации ч.д.а.

Для осуществления экстракции в делительную воронку вносили 3,0 г сульфата аммония, 4,0 мл раствора неонола АФ 9-12 с концентрацией 250 г/л, 2,0 мл 0,1 моль/л раствора соли металла, создавали необходимую кислотность введением 5 моль/л раствора хлороводородной или серной кислоты, добавляли расчетное количество 2 моль/л раствора комплексообразователя (йодида, бромида или тиоцианата калия) и доводили объем дистиллированной водой до 20 мл. Полученную смесь встряхивали до полного растворения соли, после установления равновесия водную фазу отделяли и определяли содержание катиона металла комплексонометрическим титрованием. Экспериментально установлено, что остаточное содержание ПАВ в рафинате не влияет на результаты определения. Эффективность экстракции оценивали, вычисляя степень извлечения (R, \%).

\section{Результаты и их обсуждение}

Фазовые равновесия и оптимальные параметры осуществления экстракции в системе вода - сульфат аммония - неонол АФ 9-12 при $25^{\circ} \mathrm{C}$ установлены ранее [25]. Показано, что расслаивание в системе сохраняется при кислотности менее 0,5 моль/л хлороводородной и 0,8 моль/л серной кислот, что несколько ограничивает экстракционные возможности системы в отношении экстракции галогенидных ацидокомплексов металлов, в связи с чем выбор металлов обусловлен возможностью их извлечения при невысокой кислотности.

Исследовано распределение ионов таллия (III), железа (III), индия и галлия в присутствии галогенид-ионов в системе вода - сульфат аммония - неонол АФ 9-12 (рис. 1). В качестве источника хлорид-ионов использовали хлороводородную кислоту, экстракцию бромидных и иодидных ацидокомплексов изучали, используя смесь соответствующего галогенида калия и серной кислоты. Установлено, что экстракция таллия (III) во всех случаях является количественной при содержании кислоты более 0,1 моль/л. Извлечение остальных 


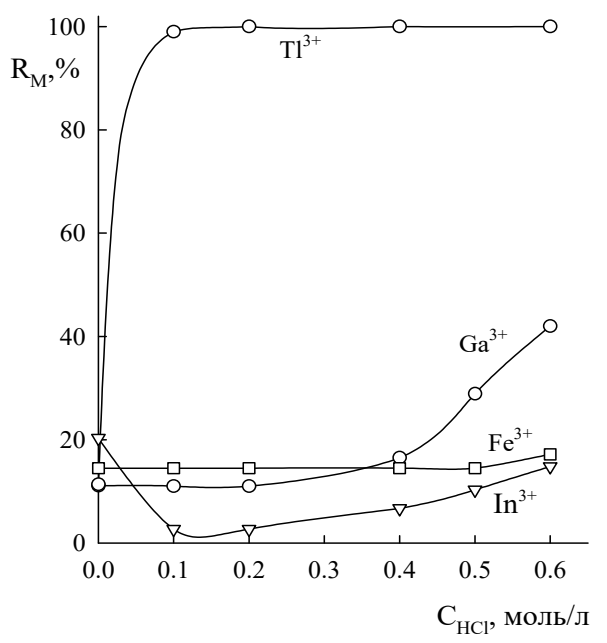

a

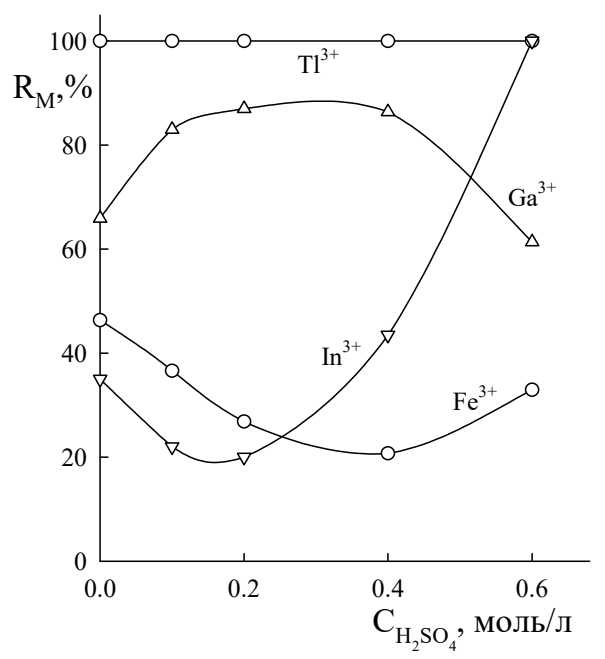

б

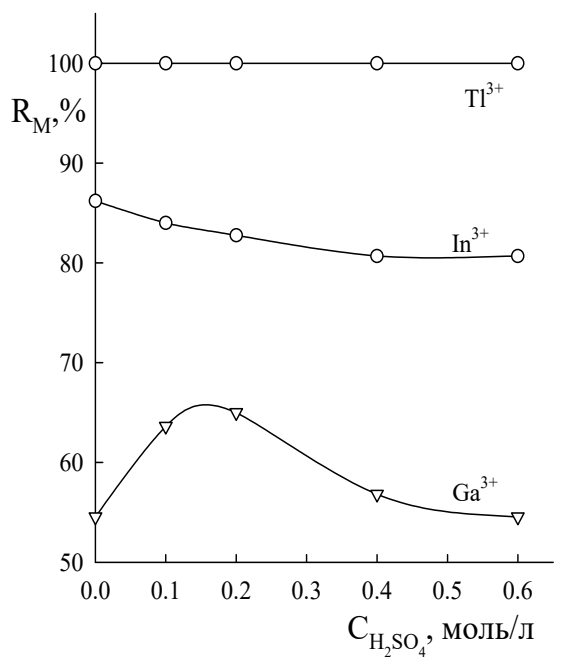

B

Рис. 1. Экстракция хлоридных (а), бромидных (б) и иодидных (в) комплексов металлов в системе вода неонол АФ 9-12 - $\left(\mathrm{NH}_{4}\right)_{2} \mathrm{SO}_{4}$ при $25{ }^{\circ} \mathrm{C}\left(\mathrm{M}^{\mathrm{n}+}-0,01\right.$ моль/л, $\mathrm{KBr}$ или $\mathrm{KI}-0,5$ моль/л, $\left(\mathrm{NH}_{4}\right)_{2} \mathrm{SO}_{4}-150,0$ г/л, неонол АФ 9-12 - 50,0 г/л)

Fig. 1. Extraction of chloride (a), bromide (b) and iodide (c) metal complexes in the water - neonol AF 9-12 $\left(\mathrm{NH}_{4}\right)_{2} \mathrm{SO}_{4}$ system at $25^{\circ} \mathrm{C}\left(\mathrm{M}^{\mathrm{n}+}-0.01 \mathrm{~mol} / 1, \mathrm{KBr}\right.$ or $\mathrm{KI}-0.5 \mathrm{~mol} / 1,\left(\mathrm{NH}_{4}\right)_{2} \mathrm{SO}_{4}-150.0 \mathrm{~g} / 1$, neonol AF 9-12 $50.0 \mathrm{~g} / \mathrm{l})$

исследованных металлов неколичественное. Степень извлечения железа (III) увеличивается при переходе от хлоридных к бромидным комплексам. Изучение распределения железа (III) в присутствии иодид-ионов не представляется возможным вследствие его восстановления.

Экстракция индия увеличивается от хлоридных к иодидным растворам, что соответствует закономерностям извлечения галогенидных комплексов нейтральными кислородсодержащими органическими растворителями [26]. Степень извлечения галлия увеличивается при переходе от хлоридных растворов к бромидным. Экстракция кадмия, висмута (III) и свинца 


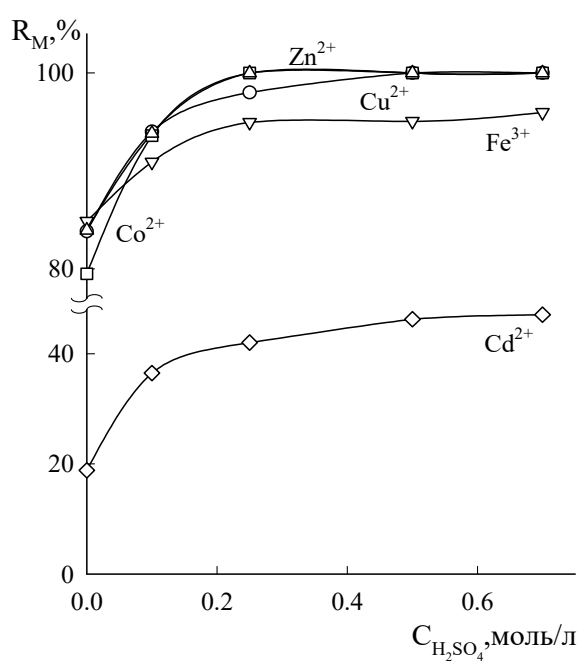

Рис. 2. Экстракция тиоцианатных комплексов металлов в системе вода - неонол АФ 9-12 - $\left(\mathrm{NH}_{4}\right)_{2} \mathrm{SO}_{4}$ при $25^{\circ} \mathrm{C}\left(\mathrm{M}^{\mathrm{n}+}-0,01\right.$ моль/л, $\mathrm{KSCN}-0,5$ моль/л, $\left(\mathrm{NH}_{4}\right)_{2} \mathrm{SO}_{4}-150,0$ г/л, неонол АФ 9-12 - 50,0 г/л)

Fig. 2. Extraction of thiocyanate metal complexes in the water - neonol AF 9-12- $\left(\mathrm{NH}_{4}\right)_{2} \mathrm{SO}_{4}$ system at $25^{\circ} \mathrm{C}$ $\left(\mathrm{M}^{\mathrm{n}+}-0.01 \mathrm{~mol} / \mathrm{l}, \mathrm{KSCN}-0.5 \mathrm{~mol} / \mathrm{l},\left(\mathrm{NH}_{4}\right)_{2} \mathrm{SO}_{4}-150.0 \mathrm{~g} / 1\right.$, neonol AF 9-12-50.0 g/l)

из иодидных растворов оказалась невозможной вследствие образования труднорастворимых иодидов и исчезновения расслаивания в системе.

Таллий (III) концентрируется в виде комплексной кислоты, при этом неонол АФ 9-12 выступает аналогом нейтрального кислородсодержащего экстрагента:

$$
\mathrm{L}_{(\mathrm{o})}+\mathrm{nH}_{(\mathrm{B})}+\mathrm{n}\left[\mathrm{TlHal}_{4}\right]_{(\mathrm{B})}^{-}=\mathrm{L}\left(\mathrm{HTlHal}_{4}\right)_{\mathrm{n}(\mathrm{o})}
$$

где L - молекула неонола АФ 9-12; Нal - галогенид-ион.

Вид кривых извлечения остальных металлов свидетельствует о сложном характере взаимодействия в экстракционной системе и наличии конкурирующих процессов, например образования сульфатных анионных комплексов.

Высокая комплексообразующая способность тиоцианат-ионов обуславливает их широкое применение при экстракции ионов металлов в расслаивающихся системах на основе водорастворимых полимеров и поверхностно-активных веществ. Введение в систему тиоцианата калия в концентрации до 0,25 моль/л значительно увеличивает степень извлечения ионов металлов. При этом количественно извлекается цинк при концентрации тиоцианат-ионов более 0,1 моль/л, кобальт (II) - более 0,15 моль/л, медь (II) - более 0,2 моль/л, железо (III) - 0,25 моль/л. Максимальная степень извлечения кадмия составляет $69 \%$.

В присутствии 0,2 моль/л тиоцианата калия в системе вода - неонол АФ 9-12 - сульфат аммония наблюдается количественное извлечение кобальта и цинка при содержании серной кислоты более 0,25 моль/л, меди (II) - более 0,5 моль/л (рис. 2). Максимальная степень извлечения железа (III) - 90 \%, а кадмия не превышает 50 \%, что объясняется низкой устойчивостью образующегося комплекса. Образующиеся при экстракции окрашенные комплексные соединения позволяют использовать исследованную систему для экстракционно-спектрофотометрического определения микроколичеств ионов металлов. 


\section{Заключение}

Оксиэтилированные нонилфенолы можно рассматривать как нейтральные кислородсодержащие экстрагенты, способные экстрагировать ионы металлов в виде галогенидных и тиоцианатных ацидокомплексов, однако узкий интервал кислотности, в котором существует расслаивание, позволяет концентрировать ограниченный перечень металлов. В системе вода - неонол АФ-9-12 - сульфат аммония наблюдается количественное концентрирование галогенидных ацидокомплексов таллия (III), а также тиоцианатных ацидокомплексов цинка, меди (II) и кобальта. Общие закономерности изменения экстрагируемости галогенидных ацидокомплексов ионов металлов позволяют говорить о подобном механизме их извлечения как органическим растворителем, так и поверхностно-активными веществами.

Предложенные системы могут использоваться для предварительного концентрирования ионов металлов с последующим их атомно-абсорбционным, атомно-эмиссионым или спектрофотометрическим (в случае экстракции с тиоцианатом калия) методами.

Работа выполнена при финансовой поддержке Министерства образования и науки Российской Федерации (задание 5.6881.2017/8.9).

\section{Список литературы}

1. Maturana H.A. Maturana H.A., Perič I.M., Pooley S.A., Rivas B.L. Poly(N-vinylpyrrolidone) as metal ion liquid-liquid extractant. Polymer Bulletin 2000. Vol. 45 (4-5), P. 425-429.

2. Bulgariu L., Bulgariu D. Extraction of metal ions in aqueous polyethylene glycol-inorganic salt two-phase systems in the presence of inorganic extractants: Correlation between extraction behaviour and stability constants of extracted species. Journal of Chromatography A 2008. Vol. 1196, P. 117124.

3. Shibukawa M. Nakayama N., Hayashi T., Shibuya D., Endo Y., Kawamura S. Extraction behaviour of metal ions in aqueous polyethylene glycol-sodium sulphate two-phase systems in the presence of iodide and thiocyanate ions. Analitical Chimica Acta 2001. Vol. 427 (2), P. 293-300.

4. Pytlakowska K., Kozik V., Dabioch M. Complex-forming organic ligands in cloud-point extraction of metal ions: a review. Talanta 2013. V. 110, P. 202-228.

5. Khan M. Khan M., Kazi T.G., Afridi H.I., Bilal M., Akhtar A., Ullah N., Talpur S. Application of ultrasonically modified cloud point extraction method for simultaneous enrichment of cadmium and lead in sera of different types of gallstone patients. Ultrasonics Sonochemistry 2017. Vol. 39, P. 313-320.

6. Luo X. Zheng H., Zhang Z., Wang M., Yang B., Huang L., Wang M. Cloud point extraction for simultaneous determination of 12 phenolic compounds by high performance liquid chromatography with fluorescence detection. Microchemical Journal 2018. Vol. 137, P. 148-154.

7. Zarrougui R., Mdimagh R., Raouafi N. Highly efficient extraction and selective separation of uranium (VI) from transition metals using new class of undiluted ionic liquids based on H-phosphonate anions. Journal of Hazardous Materials 2018. Vol. 342, P. 464-476.

8. Wei W., Cho C.W., Kim S., Song M.H., Bediako J.K., Yun Y.S. Selective recovery of Au (III), $\mathrm{Pt}$ (IV), and Pd (II) from aqueous solutions by liquid-liquid extraction using ionic liquid Aliquat-336. Journal of Molecular Liquids 2016. Vol. 216, P. 18-24. 
9. Parmentier D. Vander Hoogerstraete T., Metz S.J., Binnemans K., Kroon M.C. Selective extraction of metals from chloride solutions with the tetraoctylphosphonium oleate ionic liquid. Ind. Eng. Chem. Res. 2015. V. 54(18), P. 5149-5158.

10. Mukhopadhyay S. Mukherjee S., Adnan N.F., Hayyan A., Hayyan M., Hashim M.A., Gupta B.S. Ammonium-based deep eutectic solvents as novel soil washing agent for lead removal. Chemical Engineering Journal 2016. Vol. 294, P. 316-322.

11. Tang B., Zhang H., Row K.H. Application of deep eutectic solvents in the extraction and separation of target compounds from various samples. Journal of Separation Science 2015. Vol. 38 (6), P. 1053-1064.

12. Shishov A., Bulatov A., Locatelli M., Carradori S., Andruch V. Application of deep eutectic solvents in analytical chemistry. A review. Microchemical Journal 2017. Vol. 135, P. 33-38.

13. Santarossa D.G., Talio M.C., Fernández L.P. Aluminium traces determination in biological and water samples using a novel extraction scheme combined with molecular fluorescence. Microchemical Journal 2016. Vol. 129, P. 274-280.

14. Elokhov A.M., Kudryashova O.S., Lesnov A.E. Potential of Magnesium Salt Monoalkylpolyethylene Glycol - Water Systems for Use in Micellar Extraction. Russian Journal of Inorganic Chemistry 2016. Vol. 61 (2), P. 243-249.

15.AklZ.F.Micelle-mediated preconcentration using cationic surfactants for thespectrophotometric determination of uranium in aqueous solutions. Journal of Radioanalytical and Nuclear Chemistry 2016. Vol. 308 (2), P. 693-700.

16. Kudryashova O.S., Bortnik K.A., Denisova S.A., Chukhlantseva E.Yu., Lesnov A.E. Solubility in the water - catamine AB - (alkali metal or ammonium chloride) systems. Russian Journal of Inorganic Chemistry 2013. Vol. 58 (2), P. 250-252.

17. Hryniewicka M., Starczewska B., Syperek I. Micellar extractions with anionic surfactant SDS and the mixture of SDS/OSAS for determination lovastatin in river samples. Journal of Molecular Liquids 2013. Vol. 187, P. 320-325.

18. Denisova S.A., Lesnov A.E., Ostanina N.N. Extraction of metals in a water - potassium bis(alkylpolyoxyethylene)phosphate - ammonium sulfate system with various photometric reagents. Journal of Analytical Chemistry 2018. Vol. 73 (5), P. 427-431.

19. Chen D., Zhang P., Li Y., Mei Zh., Xiao Yu. Hexafluoroisopropanol-induced coacervation in aqueous mixed systems of cationic and anionic surfactants for the extraction of sulfonamides in water samples. Analytical and Bioanalytical Chemistry 2014. Vol. 406, P. 6051-6060.

20. Elokhov A.M., Bogomolov N.V., Denisova S.A., Kudryashova O.S., Lesnov A.E. Phase equilibria and extraction of metal cations in the systems based on synergistic mixtures of potassium bis(alkylpolyoxyethylene)phosphate and alkylbenzyldimethylammonium chloride. Russian Chemical Bulletin Vol. 67 (2), P. 206-210.

21. Lesnov A.E., Golovkina A.V., Kudryashova O.S., Denisova S.A. Phase and extraction equilibria in water - polyetheleneglycol ethers of monoethanolamides of synthetic acid - ammonium chloride systems. Russian Journal of Physical Chemistry A 2016. Vol. 90 (8), P. 1585-1589.

22. Stankova A.V., Elokhov A.M., Denisova S.A., Kudryashova O.S., Lesnov A.E. Specific Features of the Salting-out of Oxyethylated Nonylphenols Using Inorganic Salts at $25^{\circ} \mathrm{C}$. Russian Journal of Physical Chemistry A 2017. Vol. 91 (5), P. 880-886. 
23. Stankova A.V., Elokhov A.M., Kudryashova O.S. Salting-out Ability of Inorganic Salts in Solutions of Ethoxylated Nonylphenols. Russian Journal of Physical Chemistry A 2018. Vol. 92 (7), P. 1386-1391.

24. Stankova A.V., Elokhov A.M., Denisova S.A., Lesnov A.E. Extraction of chloride acido complexes of triply charged metal cations in water - oxyethylated nonylphenol - salting-out agent systems. Russian Chemical Bulletin 2018. Vol. 67 (9), P. 1608-1611.

25. Станкова А.В., Елохов А.М., Катаева Д.А. Растворимость в системах вода - оксиэтилированный нонилфенол - $\left(\mathrm{NH}_{4}\right)_{2} \mathrm{SO}_{4}$. Вестник ЮУрГУ. Серия Химия 2018. Т. 10 (3), С. 37-45 [Stankova A.V., Elokhov A.M., Kataeva D.A. Solubility in systems water - ethoxylated nonylphenol $\left(\mathrm{NH}_{4}\right)_{2} \mathrm{SO}_{4}$. SUSU Bulletin. Chemistry series 2018. Vol. 10 (3), P. 37-45 (In Russ.)]

26. Золотов Ю.А., Иофа Б.З., Чучалин Л.К. Экстракция галогенидных комплексов металлов. М.: Наука, 1973. 380 с. [Zolotov Yu.A., Iofa B.Z., Chuchalin L.K. Extraction of metal halide complexes. M., Nauka, 1973. 380 p. (In Russ.)] 